\title{
Exact Coupled-Mode Theories for Multilayer Interference Coatings with Arbitrary Strong Index Modulations
}

\author{
Nicolai Matuschek, Franz X. Kärtner, and Ursula Keller, Member, IEEE
}

\begin{abstract}
The analysis of multilayered interference coatings by coupled-mode theory is considered to be an approximation useful for small index, gain, and/or loss modulations. In this paper, we show that an exact analysis of a multilayer coating with coupledmode theory is possible by redefining the coupling and detuning coefficients. We derive the correct coefficients for the case of a Bragg mirror consisting of layers with arbitrary high and low refractive indexes. A detailed comparison with coupled-mode theories using the standard coupling and detuning coefficients is presented.
\end{abstract}

Index Terms-Coatings, coupled mode analysis, coupled transmission lines, dielectric films, electromagnetic coupling, thin film devices, transmission line theory.

\section{INTRODUCTION}

$\mathbf{F}$ OR THE DESIGN of optical filters and mirrors that are composed of high and low index layers, one usually starts from a standard structure known to solve a given design problem (see, for example, [1]). The design goal is further approached by computer optimization of this standard structure. Alternatively, there exist analytic methods for the synthesis of waveguide filters based on coupled-mode theory [2], [3]. These waveguide filters are made by small index variations in the guiding layers that result only in small reflections within a distance of one wavelength. For this case, coupled-mode theory is an excellent approximation to the exact problem [4].

It would be very useful if the analytic design tools developed for waveguide filters that are based on coupled-mode theory could be also used for broad-band filters and mirrors composed of high and low index layers, such as the chirped mirrors, invented by Szipöcs et al. [5]. One finds, for broad-band quarter-wave Bragg mirrors that consist of layer pairs with a large refractive-index difference, that coupled-mode analysis still agrees surprisingly well with the exact analysis [6].

In this paper, we show that coupled-mode theory for the description of a Bragg mirror is indeed exact for arbitrary refractive-index differences if redefined coupling and detuning coefficients are used. This explains the good agreement between normal coupled-mode theory and exact analysis for the

Manuscript received July 23, 1996; revised November 5, 1996. This work was supported by the Swiss National Science Foundation.

The authors are with the Institute of Quantum Electronics, Swiss Federal Institute of Technology, ETH-Hönggerberg HPT E-9, CH-8093 Zürich, Switzerland.

Publisher Item Identifier S 0018-9197(97)01575-3. quarter-wave Bragg mirrors with large index discontinuities as mentioned above. In the future, one can use the coupled-mode description of multilayer structures derived here to develop new design methods for multilayer coatings similar to those put forward by Matsuhara et al. [3] based on coupled-mode theory.

In Section II, we briefly review the coupled-mode theory for a periodic Bragg grating and state its transfer matrix in order to clarify notation. The exact transfer matrix of the Bragg grating is given in Section III. Its structural equivalence to the coupled-mode result is shown. Both transfer matrices are absolutely equivalent over the whole wavelength range if the coupling and detuning coefficients are properly chosen, as derived in Section IV. In contrast to the normal coupling and detuning coefficients, these redefined quantities exhibit a complicated wavelength dependence. However, with these redefined quantities, the coupled-mode analysis is exact and not limited to small index differences. In Section V, we discuss various approximations to the exact coupled-mode analysis that are normally used in the literature.

\section{COUPLED-Mode TheORY FOR A BRAGG Grating}

We consider a Bragg grating which consists of a series of homogeneous layer pairs with refractive indexes $n_{h}$ and $n_{l}$. The period of the resulting index profile, shown in Fig. 1, is given by $\Lambda=d_{h}+d_{l}$ and the effective refractive index $n_{\mathrm{eff}}$ is determined by

$$
\Lambda n_{\mathrm{eff}}=n_{h} d_{h}+n_{l} d_{l}=d_{\mathrm{opt}}
$$

where $d_{\text {opt }}$ is the optical thickness of a unit cell of the structure. In this paper, we assume that the medium is free of absorption. We only consider propagation perpendicular to the layers and describe the amplitudes of the right- and leftward traveling waves by $A(z)$ and $B(z)$, respectively. These amplitudes are normalized so that their absolute square is proportional to the energy flux in the corresponding direction. If the refractive-index difference is small, it is well known that the dynamics of the right- and leftward wave can be approximately described by the coupled-mode equation [7], [8]

$$
\frac{d}{d z}\left(\begin{array}{l}
A(z) \\
B(z)
\end{array}\right)=\left(\begin{array}{cc}
-i \beta & -i \kappa e^{-i K z} \\
i \kappa e^{i K z} & i \beta
\end{array}\right)\left(\begin{array}{l}
A(z) \\
B(z)
\end{array}\right) .
$$




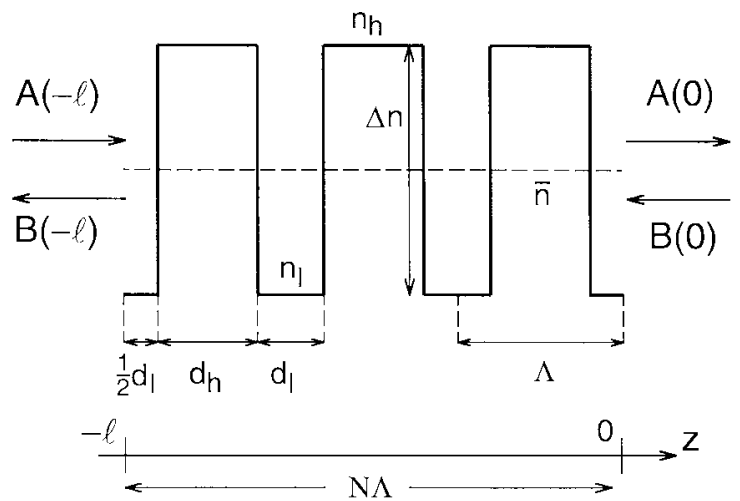

Fig. 1. Rectangular-shaped index profile with an average refractive index $\bar{n}=\frac{1}{2}\left(n_{h}+n_{l}\right)$ and an index difference $\Delta n=n_{h}-n_{l}$ for a layered media. Three periods are shown.

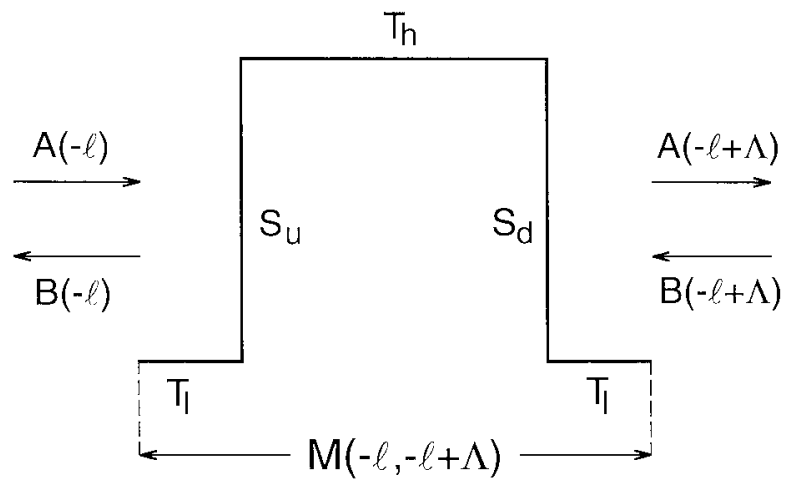

Fig. 2. The total transfer matrix for one period is written as the product of five matrices for this symmetrical definition of the unit cell. It involves three propagation steps and two jump steps (see Appendix A).

Here, the quantity $K=\frac{2 \pi}{\Lambda}$ denotes the wavenumber of the periodic index profile and $\beta=k n_{\mathrm{eff}}$ is the effective wavenumber in the layered medium, with $k=\frac{2 \pi}{\lambda}$ representing the vacuum wavenumber. In standard coupled-mode theory, the coupling coefficient $\kappa$ is determined by the first Fourier coefficient of the periodic index profile. If we choose the period of the index profile symmetrically, as shown in Fig. 2, then the coupling coefficient in (2) is a real negative number. Otherwise, the coupling coefficient includes an additional complex phase factor [6], [8].

For the rectangular-shaped index profile, we obtain with $\Delta n=n_{h}-n_{l}$ and $\bar{n}=\frac{1}{2}\left(n_{h}+n_{l}\right)[8]:$

$$
\kappa=-\frac{2 \Delta n \bar{n}}{n_{\mathrm{eff}}} \sin \left(\frac{1}{2} K d_{h}\right) \frac{1}{\lambda}<0 .
$$

If we evaluate (3) at the Bragg wavelength $\lambda_{\mathrm{B}}=2 n_{\mathrm{eff}} \Lambda$, this coupling coefficient simplifies to

$$
\kappa=-\frac{\Delta n \bar{n}}{n_{\mathrm{eff}}^{2} \Lambda} \sin \left(\frac{1}{2} K d_{h}\right) .
$$

For small index differences, quarter-wave layers and a wavelength close to the stop band around the Bragg wavelength, the coupling coefficient (4) is approximately given by [7]

$$
\kappa=-\frac{\Delta n}{\bar{n} \Lambda} \text {. }
$$

Solution of the coupled-mode equations between the positions $z=-\ell$ and $z=0$ results in the transfer matrix $\mathbf{M}(-\ell, 0)$ for the Bragg grating [6]

$$
\left(\begin{array}{l}
A(-\ell) \\
B(-\ell)
\end{array}\right)=\mathbf{M}(-\ell, 0)\left(\begin{array}{l}
A(0) \\
B(0)
\end{array}\right)
$$

with (7), shown at the bottom of the page. Here, $\ell=\Lambda N$ is the length of the medium expressed by the number of layer pairs $N$. The detuning coefficient is given by $\delta$ which describes the phase mismatch between the effective wavenumber and the half wavenumber of the index profile

$$
\delta=\beta-\frac{K}{2}=\beta-\frac{\pi}{\Lambda}
$$

and $\gamma=\sqrt{\kappa^{2}-\delta^{2}}$ is the propagation constant in the Bragg medium. Note that this propagation constant becomes purely imaginary outside the stop band, i.e., $|\kappa|<|\delta|$.

The amplitude reflectivity $r_{M}$ of the periodic structure, for a wave incident from the right, is then given by

$$
r_{\mathrm{M}}=\frac{A(0)}{B(0)}=-\frac{m_{12}}{m_{11}}
$$

where $m_{i j}$ are the matrix elements of the corresponding transfer matrix, given in this case by (7).

\section{EXACt TRANSFER MATRIX OF A BRAGG GRATING FOR ARBITRARY INDEX DIFFERENCES}

In this section, we derive a compact expression for the exact transfer matrix of a Bragg grating. The transfer matrix of one Bragg period, as shown in Fig. 2, can be obtained by the transfer matrix method [1]. For more details, see Appendix A. The result is

$$
\mathbf{M}(-\ell,-\ell+\Lambda)=\left(\begin{array}{ll}
F & G^{*} \\
G & F^{*}
\end{array}\right)
$$

with

$$
\begin{aligned}
F & =\frac{1}{1-r^{2}}\left(e^{i \phi}-r^{2} e^{-i \Delta \phi}\right) \\
G & =\frac{-2 i r}{1-r^{2}} \sin \left(\phi_{h}\right) \\
\phi & =\phi_{h}+\phi_{l} \\
\Delta \phi & =\phi_{h}-\phi_{l} \\
\phi_{h} & =k n_{h} d_{h} \\
\phi_{l} & =k n_{l} d_{l} \\
r & =\frac{\Delta n}{2 \bar{n}}
\end{aligned}
$$

$$
\mathbf{M}(-\ell, 0)=(-1)^{N}\left(\begin{array}{cc}
\cosh (\gamma \Lambda N)+i \frac{\delta}{\gamma} \sinh (\gamma \Lambda N) & i \frac{\kappa}{\gamma} \sinh (\gamma \Lambda N) \\
-i \frac{\kappa}{\gamma} \sinh (\gamma \Lambda N) & \cosh (\gamma \Lambda N)-i \frac{\delta}{\gamma} \sinh (\gamma \Lambda N)
\end{array}\right)
$$


and $F^{*}$ and $G^{*}$ denote the complex conjugate quantities. Note that the particular structure of matrix (10) combined with the unimodularity

$$
\operatorname{det} \mathbf{M}(-\ell,-\ell+\Lambda)=|F|^{2}-|G|^{2}=+1
$$

is a consequence of the losslessness (energy conservation) and time-reversal invariance of the structure, as is shown in Appendix B.

Since the total grating is a sequence of $N$ unit cells with transfer matrix (10), the transfer matrix of the periodic structure is given as the $N$ th power of this matrix, i.e., $\mathbf{M}(-\ell, 0)=\mathbf{M}^{N}(-\ell,-\ell+\Lambda)$. To calculate this expression in a simple way, one needs to know the eigenvalues of matrix (10) that determine the behavior of the field amplitudes. The two eigenvalues are

$$
\lambda_{1,2}=F_{R} \mp \sqrt{F_{R}^{2}-1}
$$

with

$$
F_{R}=\Re\{F\}=\frac{1}{1-r^{2}}\left(\cos (\phi)-r^{2} \cos (\Delta \phi)\right)
$$

and

$$
F_{I}=\Im\{F\}=\frac{1}{1-r^{2}}\left(\sin (\phi)+r^{2} \sin (\Delta \phi)\right)
$$

where we have used the identity

$$
\sqrt{F_{R}^{2}-1}=\sqrt{|G|^{2}-F_{I}^{2}}
$$

which follows from (18). Because of the unimodularity and time-reversal invariance of the transfer matrix, its eigenvalues (19) fulfill the conditions

$$
\lambda_{1} \cdot \lambda_{2}=1 \quad \text { and } \quad \lambda_{1}+\lambda_{2} \in \mathbb{R} .
$$

Thus, only two cases are possible:

I) $\lambda_{1}, \lambda_{2} \in \mathbb{R} \Leftrightarrow\left|F_{R}\right| \geq 1$ : The eigenvalues are real and can be written as

$$
\lambda_{1}=\mp e^{\eta}, \quad \lambda_{2}=\mp e^{-\eta}, \quad \eta \in \mathbb{R}^{+} .
$$

The field in the grating then exponentially increases or decreases, respectively. This wavelength range corresponds to the stop band of the Bragg grating.

II) $\lambda_{1}, \lambda_{2} \in \mathbb{C} \Leftrightarrow\left|F_{R}\right|<1$ : The eigenvalues can be written as

$$
\lambda_{1}=\mp e^{i \eta}, \quad \lambda_{2}=\mp e^{-i \eta}, \quad \eta \in \mathbb{R}^{+}
$$

and the field shows an oscillating behavior. This wavelength range corresponds to the transmission band of the Bragg grating.

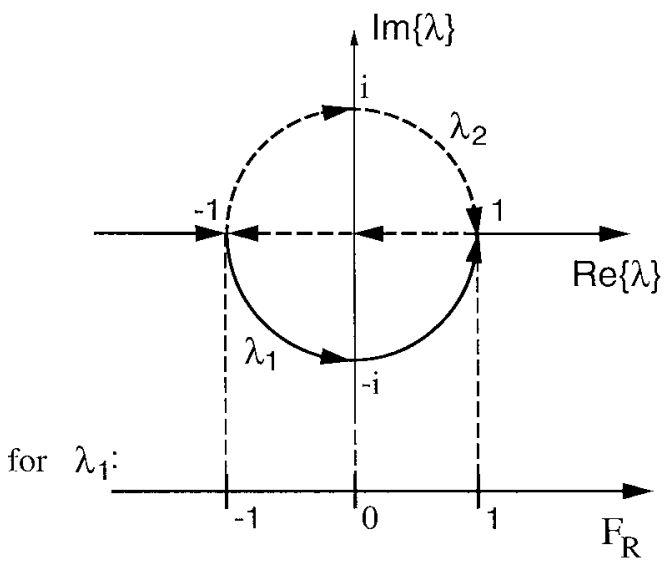

Fig. 3. Loci of the roots $\lambda_{1}$ and $\lambda_{2}$ in the complex $\lambda$ plane. Each arrow represents one of the four ranges of $F_{R}$. In this figure, the $F_{R}$ axis corresponds to the eigenvalue $\lambda_{1}$. Note that $\lambda_{2}$ is the reciprocal of $\lambda_{1}$.

To avoid ambiguities, we cover all cases by $\lambda_{1}=-e^{\eta}=$ $e^{\eta-i \pi}$, where $\eta \in \mathbb{C}$ is piecewise defined as

$$
\eta=\left\{\begin{array}{ll}
\ln \left(-F_{R}+\sqrt{F_{R}^{2}-1}\right) ; & F_{R} \leq-1 \\
i \arctan \left(\frac{\sqrt{1-F_{R}^{2}}}{-F_{R}}\right) ; & -1<F_{R} \leq 0 \\
-i \arctan \left(\frac{\sqrt{1-F_{R}^{2}}}{F_{R}}\right)+i \pi ; & 0<F_{R} \leq+1 \\
\ln \left(F_{R}-\sqrt{F_{R}^{2}-1}\right)+i \pi ; & F_{R}>+1
\end{array} .\right.
$$

Note that with this definition, the first case describes the usual stop band regime near the Bragg wavelength $\lambda_{\mathrm{B}}$. For this range, $\eta$ is a real positive number. The fourth case also describes a stop band that can only occur if $\Delta \phi \neq 0$, because otherwise the condition $F_{R}>1$ cannot be satisfied according to (20). The appearance of this additional stop band will be discussed in detail in Section V. As an illustration, Fig. 3 shows the loci of the roots $\lambda_{1}$ and $\lambda_{2}$ in the $\lambda$ plane in dependence on $F_{R}$.

With $\lambda_{1}$ and $\eta$ as defined in (26), we obtain

$$
\begin{aligned}
F_{R} & =-\cosh \eta \\
\sqrt{F_{R}^{2}-1} & =\sinh \eta
\end{aligned}
$$

and the transfer matrix of a unit cell (10) can be written as (29), shown at the bottom of the page. It can be proven by induction that the $N$ th power of this unimodular matrix is given by (30), shown at the bottom of the page.

As is shown in Appendix B, all matrices of the form (10) with $\operatorname{det} \mathbf{M}=1$ generate a matrix subgroup of the special

$$
\begin{aligned}
\mathbf{M}(-\ell,-\ell+\Lambda) & =-\left(\begin{array}{cc}
\cosh \eta-i \frac{F_{I}}{\sqrt{F_{R}^{2}-1}} \sinh \eta & -\frac{G^{*}}{\sqrt{F_{R}^{2}-1}} \sinh \eta \\
-\frac{G}{\sqrt{F_{R}^{2}-1}} \sinh \eta & \cosh \eta+i \frac{F_{I}}{\sqrt{F_{R}^{2}-1}} \sinh \eta
\end{array}\right) \\
\mathbf{M}(-\ell, 0) & =M^{N}(-\ell,-\ell+\Lambda)=(-1)^{N}\left(\begin{array}{cc}
\cosh (\eta N)-i \frac{F_{I}}{\sqrt{F_{R}^{2}-1}} \sinh (\eta N) & -\frac{G^{*}}{\sqrt{F_{R}^{2}-1}} \sinh (\eta N) \\
-\frac{G}{\sqrt{F_{R}^{2}-1}} \sinh (\eta N) & \cosh (\eta N)+i \frac{F_{I}}{\sqrt{F_{R}^{2}-1}} \sinh (\eta N)
\end{array}\right)(
\end{aligned}
$$


linear group $\mathrm{SL}(2, \mathbb{C})$. For this reason, also the coupled-mode matrix (7) is an element of this subgroup. The matrix (30) must obey the power law given here, because the transfer matrix of the unit cell (29) is of the same form as the transfer matrix of the coupled-mode theory (7). Therefore, it is an element of the same subgroup of $\mathrm{SL}(2, \mathbb{C})$, and the transfer matrix of the coupled-mode theory obviously obeys this power law. It is a special case of the generally valid power law for an arbitrary unimodular matrix, as given in [9] and proven in [10].

\section{EXACT COUPLED-Mode THEORY}

Comparison of the two transfer matrices (7) and (30) shows that they are structurally equivalent and even equal, if the corresponding coefficients and arguments of the hyperbolic functions are matched. Thus, we identify the coupling and detuning coefficients of the coupled-mode equations, which give the correct transfer matrix of the Bragg grating, as

$$
\begin{aligned}
\kappa \Lambda & =-i \alpha G=-\alpha \frac{2 r}{1-r^{2}} \sin \left(\phi_{h}\right) \\
\delta \Lambda & =-\alpha F_{I}=-\alpha \frac{1}{1-r^{2}}\left(\sin (\phi)+r^{2} \sin (\Delta \phi)\right) .
\end{aligned}
$$

Here, the factor $\alpha$ has to be chosen such that the propagation constants of the exact transfer matrix and the equivalent coupled-mode matrix agree, i.e.,

$$
\gamma \Lambda=\sqrt{\kappa^{2}-\delta^{2}} \Lambda=\alpha \sqrt{F_{R}^{2}-1} \stackrel{!}{=} \eta
$$

From (33) with (28), we can obtain an explicit expression for $\alpha$ :

$$
\alpha=\frac{\eta}{\sinh \eta}
$$

where $\eta$ is determined according to (26). Thus, our conclusion is that the coupled-mode equations exactly describe a Bragg grating for arbitrary refractive-index differences, if the redefined quantities (31) and (32) are used instead of the normal values presented in Section II.

\section{COMPARISON BETWEEN EXACT AND APPROXIMATE COUPLED-MODE THEORY}

In this section, we compare the standard coupled-mode theories that use the coupling coefficients (3) or (4) and detuning coefficient (8), with the exact results (31) and (32). Our comparison is made for a mirror which consists of five periods of layers with a Bragg wavelength at $800 \mathrm{~nm}$. We choose the refractive indices $n_{l}=1.5$ and $n_{h}=2.5$, i.e., $r=0.25$, which are close to the refractive indexes of $\mathrm{SiO}_{2}$ and $\mathrm{TiO}_{2}$ used for standard laser mirrors.

First, we want to discuss the case of a quarter-wave Bragg mirror, i.e., $\Delta \phi=0\left(n_{h} d_{h}=n_{l} d_{l}=200 \mathrm{~nm}\right)$. Fig. 4 shows the numerical results for the amplitude reflectivity $r_{M}=\sqrt{R} e^{i \Phi}$. In Fig. 4(a), we show its absolute square $R$ and in Fig. 4(b) its phase $\Phi$ as a function of wavenumber normalized to the Bragg wavenumber $k_{B}=\pi /\left(\Lambda n_{\mathrm{eff}}\right)$. Four different cases were computed.

I) exact calculation, using the coupling coefficient (31) and detuning coefficient (32);

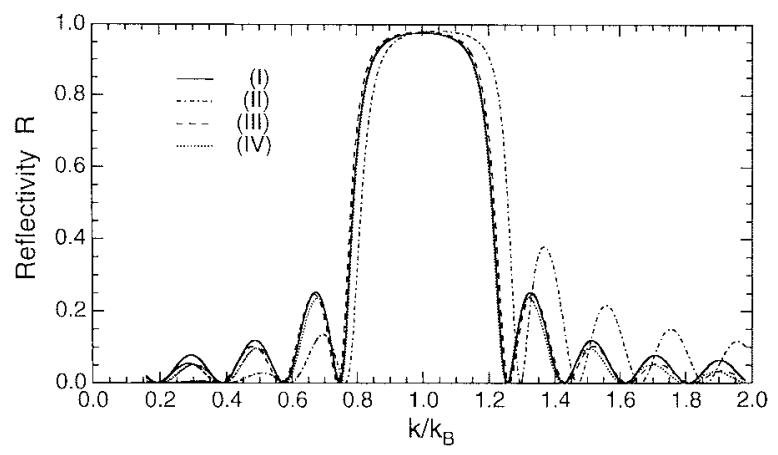

(a)

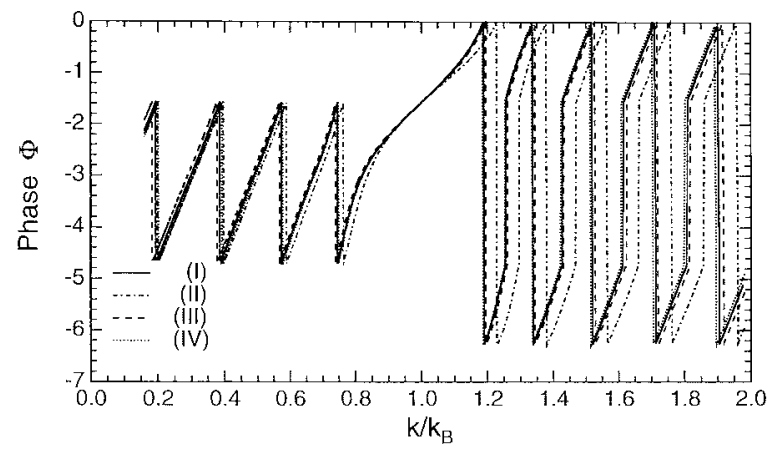

(b)

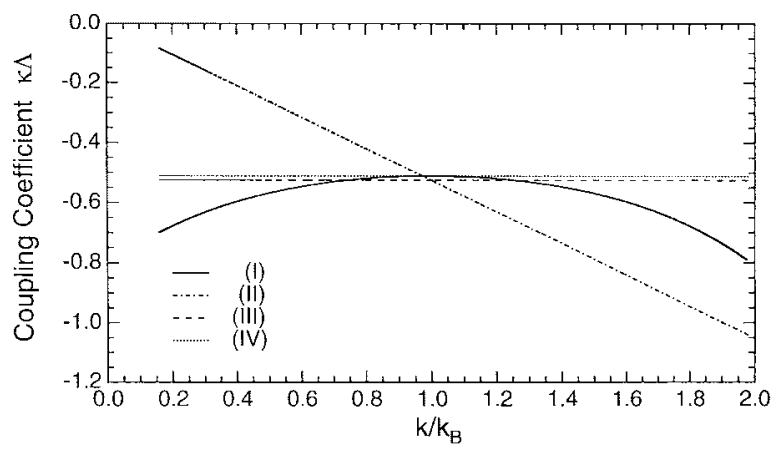

(c)

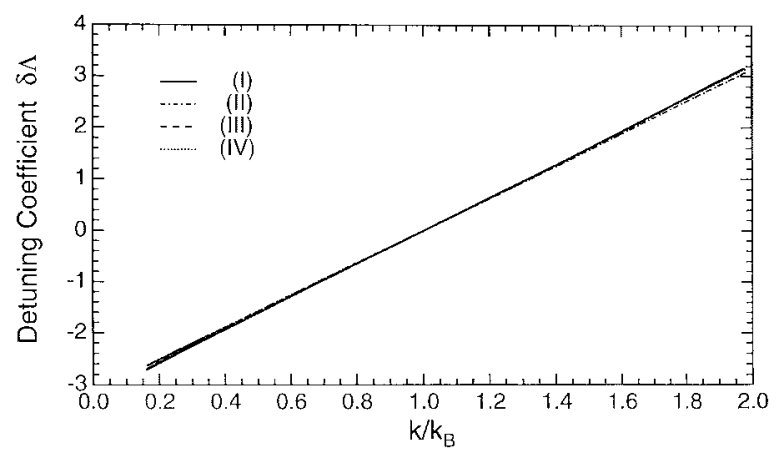

(d)

Fig. 4. Comparison of the coupled-mode results with the exact results for a Bragg mirror composed of $N=5$ layer pairs. The refractive indexes are $n_{h}=2.5$ and $n_{l}=1.5$.The thickness of the layers was chosen $d_{h}=80$ $\mathrm{nm}$ and $d_{l}=133 \mathrm{~nm}$, so that the optical thickness per layer $(200 \mathrm{~nm})$ is a quarter wavelength. We show results for (a) reflectivity, (b) phase, (c) coupling coefficient, and (d) detuning coefficient for the four different cases as defined in the text: 1) exact calculation using (31) and (32);2) the usual coupled-mode theory using (3) and (8); 3 ) the usual coupled-mode theory using (4) and (8); 4) using the linearized approximations (C-1) and (C-2) of the exact coupling and detuning coefficients. 
II) approximation, using the normal coupling coefficient (3) and detuning coefficient (8);

III) approximation, using the simplified coupling coefficient (4) and detuning coefficient (8);

IV) approximation, using a linear expansion of the redefined coupling and detuning coefficients with respect to wavenumber, as presented in Appendix C.

The cases III) and IV) agree surprisingly well with the exact result I) over the whole wavelength range shown, whereas case II), the normal coupling coefficient, results in a considerable deviation. The same is true for the phase. The reason for this behavior can be explained by Fig. 4(c) and (d) where the coupling and detuning coefficients used in the four cases are shown. For a quarter-wave stack, the exact coupling coefficient depends to lowest order only quadratic on the wavenumber around $k_{B}$ (see Appendix C). Therefore, a constant coupling coefficient leads to a better approximate description of the Bragg mirror than the value (3) derived from the Fourier expansion of the index profile. Obviously, the linear dependence of the coupling coefficient (3) on wavenumber leads to a shift of the stop band to higher wavenumbers that destroys the symmetry relative to the Bragg wavenumber. Therefore, the range to the right of $k_{B}$ is overemphasized by the larger absolute value of the coupling coefficient in this range. Fig. 4(d) shows that the discussion of the quality of various approximations essentially can be reduced to a discussion of the coupling coefficient, because all detuning coefficients show approximately the same linear behavior over the whole wavelength range depicted.

Note that the approximations 2)- 4) become arbitrarily wrong in the regime when the next stop band of the exact computation arises. The reason is that all approximations to the exact expressions are expansions around the Bragg wavelength and, therefore, only the stop band around this wavelength exists. The greater the deviation from the Bragg wavelength, the greater the phase mismatch given by the detuning coefficient $\delta$, which is responsible for the decay of the envelope of the reflectivity $R$ to zero instead of the periodic revival of the exact calculated reflectivity. In the case of $\Delta \phi=0$, the condition for a maximum reflectivity is $k / k_{B}=2 m-1$, for all positve Integers $m$, thus, the next stop band is centered at $k / k_{B}=3$ (not shown in Fig. 4). This can be explained by the fact that all waves reflected at any refractive-index step are in phase at the position $z=0$, and therefore show maximum constructive interference.

The situation becomes more complex for the case of layer pairs with unequal optical thicknesses, i.e., $\Delta \phi \neq 0$. Fig. 5 shows the results for $n_{h} d_{h}=100 \mathrm{~nm}$ and $n_{l} d_{l}=300 \mathrm{~nm}$. Due to the deviation from quarter-wave layers, the absolute value of the coupling coefficient around the Bragg wavelength is reduced [compare Figs. 4(c) and 5(c)] which also reduces the width and the peak reflectivity of the corresponding stop band [see Fig. 5(a) and (b)]. Again, the worst approximation is given for case 2), the normally used coupled-mode theory. As for the quarter-wave stack, the stop band seems to be shifted and the quality of the approximation 2) for both cases is similar. Case 3) leads again to a very accurate agreement at least in the stop band regime, although the accuracy is not as good as for the

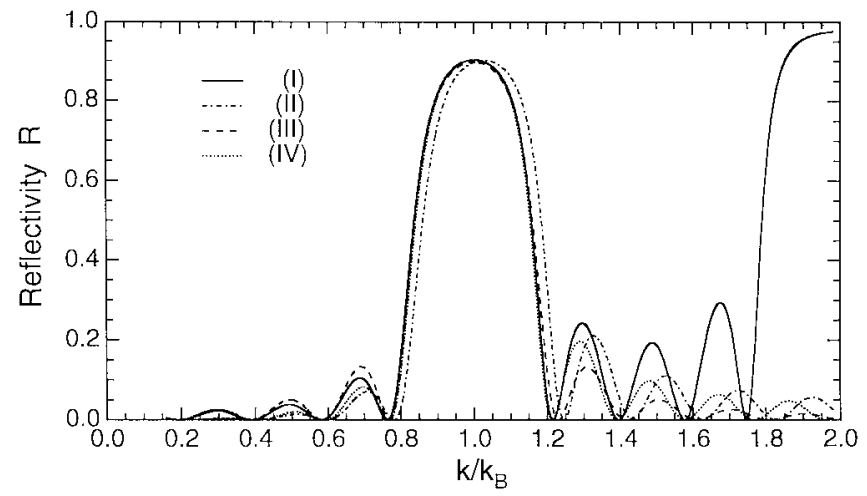

(a)

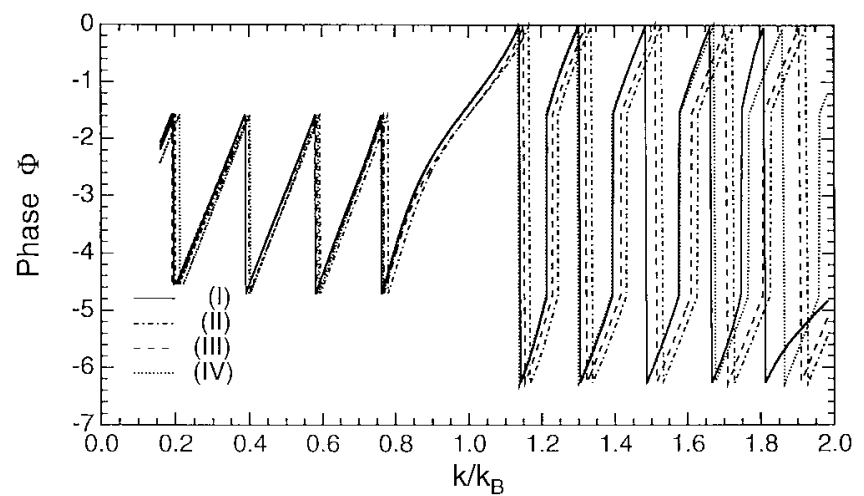

(b)

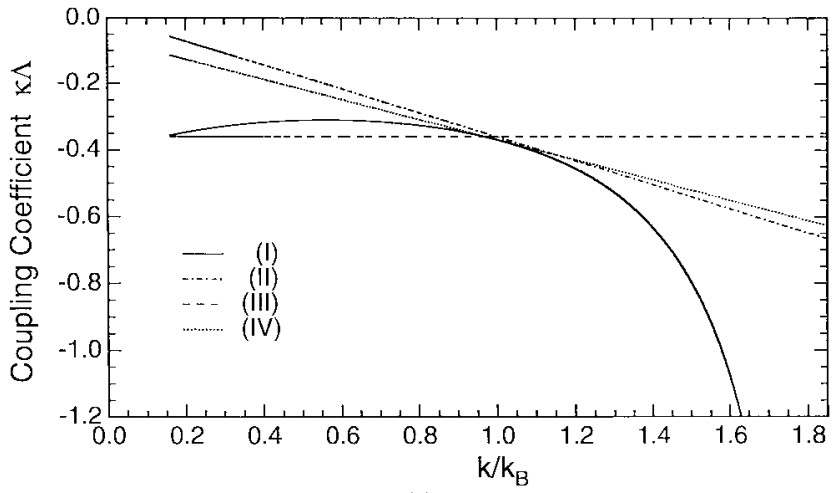

(c)

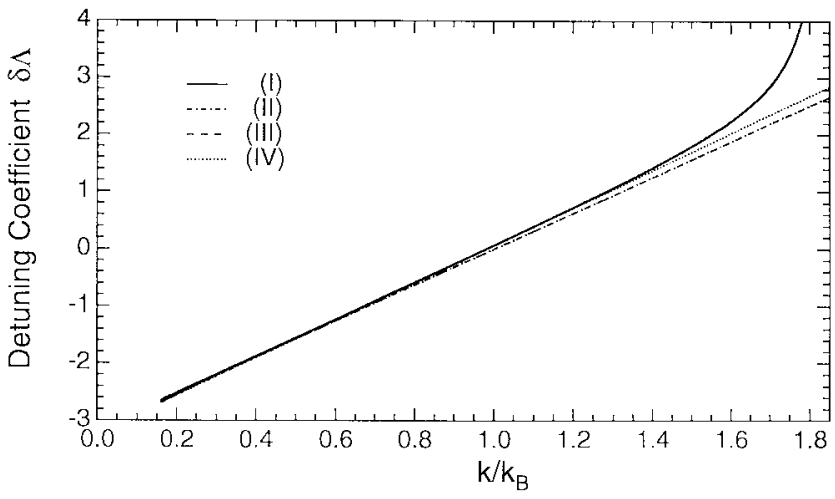

(d)

Fig. 5. Comparison of the coupled-mode results with the exact results for a Bragg mirror composed of $N=5$ layer pairs. Again the refractive indexes are $n_{h}=2.5$ and $n_{l}=1.5$. However, the thickness of the layers is now chosen $d_{h}=40 \mathrm{~nm}$ and $d_{l}=200 \mathrm{~nm}$ so that the difference in the optical thickness per period is $n_{h} d_{h}-n_{l} d_{l}=-200 \mathrm{~nm}$. We show results for (a) reflectivity, (b) phase, (c) coupling coefficient, and (d) detuning coefficient where 1), 2), 3), and 4) are the same four different cases as defined in Fig. 4. 
case of a quarter-wave stack. Again, the best approximation is the redefined coupled-mode theory with a linear approximation of the coupling and detuning coefficients, case 4).

For the case $\Delta \phi \neq 0$, the exact calculation shows a new stop band centered at $k / k_{B}=2$ with an even greater peak reflectivity than at the Bragg wavelength [see Fig. 5(a)]. For this wavenumber range, $F_{R}$ is greater than +1 and the redefined coupling and detuning coefficients of the exact coupled-mode theory become complex. Therefore, they are not any longer comparable with their real approximations shown in Fig. 5(c) and (d). Obviously, the additional stop band is responsible for the reduced agreement between the normal coupled-mode theory and the exact results in comparison to the case $\Delta \phi=0$. By increasing the deviation at the Bragg wavelength $\Delta \phi_{0}$ from its value at a quarter-wave stack $\Delta \phi_{0}=0$, the peak reflectivity of the second stop band grows, whereas the reflectivity at the Bragg wavelength decreases if $\phi_{0}=\pi$ is held constant. At $\left|\Delta \phi_{0}\right|=\frac{\pi}{2}$, the reflectivity of the stop band at $k / k_{B}=2$ reaches its maximum value. Fig. 5 shows the results for $\Delta \phi_{0}=-\frac{\pi}{2}$ and therefore depicts the worst case when compared with the exact results. The higher peak reflectivity at $2 k_{B}$ can be explained as follows: all waves reflected at any refractive-index step are in phase at the position $z=0$ if the conditions $\left|\Delta \phi_{0}\right|=\frac{\pi}{2}$ and $k / k_{B}=$ $4 m-2$ for all positive integers $m$ are fulfilled, whereas at wavenumbers defined by $k / k_{B}=2 m-1$, a partially destructive interference of the reflected waves occurs now. (In this example, for the wavenumbers defined by $k / k_{B}=4 \mathrm{~m}$, the amplitudes interference is totally destructive.)

The different quality of the approximations can be again explained by the behavior of the coupling and detuning coefficients. If the optical thicknesses of the layers are not equal $(\Delta \phi \neq 0)$, the exact coupling coefficient has also a linear term (see Appendix C). Then, both the first Fourier coefficient with the wrong slope [case 2)] and the constant approximation of the coupling coefficient [case 3)] result in a considerable deviation from the exact result. Especially, the worse agreement of case 3) directly follows from the greater deviation of the coupling coefficient, as the comparison between the Figs. 4(c) and 5(c) clearly shows. Now, also the deviation in the detuning coefficient is not negligible.

To summarize this section, for the analysis of Bragg structures with relatively high index differences, the usual coupledmode analysis leads to a surprisingly good agreement with exact calculations. This is due to the fact that there exist coupled-mode equations with special coupling and detuning coefficients which solve the problem exactly. We have found that for a Bragg mirror the constant coupling coefficient (4) leads to better results than the coupling coefficient (3) derived from the first Fourier coefficient of the grating, which is normally used.

\section{CONCLUSION}

We have studied a Bragg mirror with a rectangular-shaped index profile by coupled-mode theory and transfer matrix method. We have shown that the transfer matrices derived with both methods are structurally equivalent. It is possible to attain an absolute equivalence between both matrices by suitable definition of the coupling and detuning coefficients. Thus, there is an exact coupled-mode theory for Bragg gratings with arbitrary refractive index differences.

The prerequisite for the existence of an exact coupled-mode theory is only that the transfer matrix of a unit cell has the form stated in (10). However, Appendix B proves that this is the case for every lossless and time-reversal invariant Bragg grating with an arbitrarily shaped index profile. Thus, there always exists a set of equivalent coupled-mode equations that exactly describe a periodic Bragg structure. Here, we derived the exact coupling and detuning coefficients for the special case of a rectangular-shaped index profile by the transfer matrix method, which is the technically most important case with respect to optical coatings.

\section{APPENDIX A \\ Transfer Matrix of OnE BragG PERIOD}

The transfer matrix for the first Bragg period, which connects the amplitudes at the positions $z=-\ell$ and $z=-\ell+\Lambda$, according to Fig. 2 and (10), can be written as the following product:

$$
\mathbf{M}(-\ell,-\ell+\Lambda)=\mathbf{T}_{l} \cdot \mathbf{S}_{u} \cdot \mathbf{T}_{h} \cdot \mathbf{S}_{d} \cdot \mathbf{T}_{l}
$$

The explicit form of the propagation matrices $\mathbf{T}_{h}$ and $\mathbf{T}_{l}$ is

$$
\begin{aligned}
& \mathbf{T}_{h}=\left(\begin{array}{cc}
e^{i \phi_{h}} & 0 \\
0 & e^{-i \phi_{h}}
\end{array}\right) \\
& \mathbf{T}_{l}=\left(\begin{array}{cc}
e^{i \frac{\phi_{l}}{2}} & 0 \\
0 & e^{-i \frac{\phi_{l}}{2}}
\end{array}\right)
\end{aligned}
$$

with $\phi_{h}=k n_{h} d_{h}$ and $\phi_{l}=k n_{l} d_{l}$. The normalized Fresnel matrices which describe the refractive index jumps "up" and "down" are given by

$$
\begin{aligned}
\mathbf{S}_{u} & =\frac{1}{\sqrt{n_{h} n_{l}}}\left(\begin{array}{cc}
\bar{n} & -\frac{1}{2} \Delta n \\
-\frac{1}{2} \Delta n & \bar{n}
\end{array}\right) \\
\mathbf{S}_{d} & =\frac{1}{\sqrt{n_{h} n_{l}}}\left(\begin{array}{cc}
\bar{n} & \frac{1}{2} \Delta n \\
\frac{1}{2} \Delta n & \bar{n}
\end{array}\right) .
\end{aligned}
$$

We introduce the sum and difference of the phases $\phi=\phi_{h}+\phi_{l}$, $\Delta \phi=\phi_{h}-\phi_{l}$ and the Fresnel reflectivity $r=\frac{\Delta n}{2 \bar{n}}$ which is the reflectivity per layer. With these definitions, and multiplying the matrices according to (A1), we find

$$
\begin{gathered}
\mathbf{M}(-\ell,-\ell+\Lambda) \\
=\frac{1}{1-r^{2}}\left(\begin{array}{cc}
e^{i \phi}-r^{2} e^{-i \Delta \phi} & 2 i r \sin \left(\phi_{h}\right) \\
-2 i r \sin \left(\phi_{h}\right) & e^{-i \phi}-r^{2} e^{i \Delta \phi}
\end{array}\right) \\
\text { APPENDIX B } \\
\text { TRANSFER MATRIX IN THE CASE OF ENERGY } \\
\text { CONSERVATION AND TIME-REVERSAL INVARIANCE }
\end{gathered}
$$

We show that due to energy conservation and time-reversal invariance, an arbitrary transfer matrix $\mathbf{T}$ is necessarily an element of the matrix group $\mathrm{SL}(2, \mathbb{C})$ and possesses complex conjugate matrix elements

$$
\mathbf{T}=\left(\begin{array}{ll}
t_{11} & t_{21}^{*} \\
t_{21} & t_{11}^{*}
\end{array}\right)
$$


with

$$
\operatorname{det} \mathbf{T}=\left|t_{11}\right|^{2}-\left|t_{21}\right|^{2}=+1 .
$$

Hence, these matrices form a subgroup of the group $\mathrm{SL}(2, \mathbb{C})$.

Proof: The transfer matrix $\mathbf{T}$ combines the amplitudes of the right and left traveling waves by

$$
\left(\begin{array}{l}
A_{1} \\
B_{1}
\end{array}\right)=\mathbf{T} \cdot\left(\begin{array}{l}
A_{2} \\
B_{2}
\end{array}\right)
$$

where the matrix is of the general form

$$
\mathbf{T}=\left(\begin{array}{ll}
t_{11} & t_{12} \\
t_{21} & t_{22}
\end{array}\right)
$$

Time-reversal invariance requires that the matrix (B4) does not change under the time-reversal operation $t \rightarrow-t$ which is equivalent to the following exchange of the amplitudes:

$$
\begin{aligned}
& A_{i} \rightarrow B_{i}^{*} \\
& B_{i} \rightarrow A_{i}^{*} .
\end{aligned}
$$

Backtransformation and reordering of the original amplitudes finally leads to the matrix

$$
\mathbf{T}=\left(\begin{array}{cc}
t_{22}^{*} & t_{21}^{*} \\
t_{12}^{*} & t_{11}^{*}
\end{array}\right)
$$

Comparison of the elements of the matrix (B4) with (B7) results in the conditions

$$
\begin{aligned}
& t_{11}=t_{22}^{*} \Leftrightarrow t_{11}^{*}=t_{22} \\
& t_{12}=t_{21}^{*} \Leftrightarrow t_{12}^{*}=t_{21} .
\end{aligned}
$$

Thus, time-reversal invariance requires a matrix of the form (B1).

The unimodularity (B2) is then a consequence of energy conservation, which means that the net flow of energy, i.e., the difference of the squared absolute values of the amplitudes, has to be preserved:

$$
\left|A_{1}\right|^{2}-\left|B_{1}\right|^{2}=\left|A_{2}\right|^{2}-\left|B_{2}\right|^{2} .
$$

Substitution of (B3) into the left side of (B10) and comparison of the coefficients leads to

$$
\left|t_{11}\right|^{2}-\left|t_{21}\right|^{2}=1 \text {. }
$$

With (B8) and (B9), it follows that this expression is exactly the determinant (B2); thus, under the given prerequisites the assertions (B1) and (B2) about an arbitrary transfer matrix are proven.

The property that all possible transfer matrices (B1) generate a subgroup of the special linear group $\operatorname{SL}(2, \mathbb{C})$ follows directly from the multiplication of two arbitrary transfer matrices. The result is a new matrix which is again unimodular and of the same form as matrix (B1). Therefore, this set of matrices is closed under matrix multiplication. From the physical viewpoint, it is a necessary consequence of energy conservation and time-reversal invariance. Obviously, matrix multiplication is an associative operation. There also exists a unimodular inverse matrix to each transfer matrix of the form (B1) and the neutral element (identity) is represented by the unit matrix. Thus, all conditions that define a group (here, the subgroup of $\mathrm{SL}(2, \mathbb{C})$ ) are fulfilled, but note that this group is not abelian.

\section{APPENDIX C}

LINEARIZATION OF THE COUPLING COEFFICIENT AND DETUNING PARAMETER AT THE BRAGG WAVELENGTH

In this appendix, we expand the redefined coupling and detuning coefficients (31) and (32) in a Taylor series at the Bragg wavelength up to terms of first order and show that we get the usual expressions from Section II if we use quarterwave layers $(\Delta \phi=0)$ in the limit $r \ll 1$.

The Bragg wavelength is defined by $\phi_{0}=\pi$. So, the Taylor series at this point can be written in the form

$$
\begin{aligned}
& \kappa \Lambda=\kappa_{0} \Lambda+\kappa_{1} \Lambda(\phi-\pi)+O\left((\phi-\pi)^{2}\right) \\
& \delta \Lambda=\delta_{0} \Lambda+\delta_{1} \Lambda(\phi-\pi)+O\left((\phi-\pi)^{2}\right) .
\end{aligned}
$$

The quantities $\kappa_{0} \Lambda$ and $\delta_{0} \Lambda$ denote the exact expressions (31) and (32) for the coupling and detuning coefficients evaluated at the Bragg wavelength and $\kappa_{1} \Lambda$ and $\delta_{1} \Lambda$ are the corresponding first derivatives with respect to $\phi$. The calculation of these four values results in

$$
\begin{aligned}
\kappa_{0} \Lambda=\left.\kappa\right|_{\phi_{0}=\pi} \Lambda= & -\frac{2 r}{1-r^{2}} \alpha_{0} \sin \left(\phi_{h, 0}\right) \\
\kappa_{1} \Lambda=\left.\frac{\partial \kappa}{\partial \phi}\right|_{\phi_{0}=\pi}= & -\frac{2 r}{1-r^{2}} \\
& \left(\alpha_{0} \frac{\phi_{h, 0}}{\pi} \cos \left(\phi_{h, 0}\right)-\frac{r^{2}}{1-r^{2}} \frac{1+\alpha_{0} F_{R, 0}}{F_{R, 0}^{2}-1}\right. \\
& \left.\times \frac{\Delta \phi_{0}}{\pi} \sin \left(\Delta \phi_{0}\right) \sin \left(\phi_{h, 0}\right)\right) \quad(\mathrm{C} 4) \\
\delta_{0} \Lambda=\left.\delta\right|_{\phi_{0}=\pi} \Lambda=- & \frac{r^{2}}{1-r^{2}} \alpha_{0} \sin \left(\Delta \phi_{0}\right) \\
\delta_{1} \Lambda=\left.\frac{\partial \delta}{\partial \phi}\right|_{\phi_{0}=\pi}= & -\frac{1}{1-r^{2}} \quad(\mathrm{C} 5) \\
& \times\left(\alpha_{0}\left(-1+\frac{\Delta \phi_{0}}{\pi} r \cos \left(\Delta \phi_{0}\right)\right)\right. \\
& \left.\quad-\frac{r^{4}}{1-r^{2}} \frac{1+\alpha_{0} F_{R, 0}}{F_{R, 0}^{2}-1} \frac{\Delta \phi_{0}}{\pi} \sin \left(\Delta \phi_{0}\right)\right)
\end{aligned}
$$

with

$$
\begin{aligned}
\alpha_{0} & =\frac{\ln \left(-F_{R, 0}+\sqrt{F_{R, 0}^{2}-1}\right)}{\sqrt{F_{R, 0}^{2}-1}} \\
F_{R, 0} & =-\frac{1}{1-r^{2}}\left(1+r^{2} \cos \left(\Delta \phi_{0}\right)\right) \\
\Delta \phi_{0} & =k_{B}\left(n_{h} d_{h}-n_{l} d_{l}\right) \\
\phi_{h, 0} & =k_{B} n_{h} d_{h} .
\end{aligned}
$$

In general, none of the four expressions (C3)-(C6) vanishes. So, (C1) and (C2) can not be simplified, and the linear expressions here are very different from the simple expressions 
(3), (4), and (8) normally used. But we see directly that a simplification is possible if the layers have the same optical thickness $\left(\Delta \phi_{0}=0, \phi_{h, 0}=\phi_{l, 0}=\frac{\pi}{2}\right)$. In this case, we get

$$
\begin{aligned}
\kappa_{0} \Lambda & =-\ln \left(\frac{1+r}{1-r}\right) \\
\kappa_{1} \Lambda & =0 \\
\delta_{0} \Lambda & =0 \\
\delta_{1} \Lambda & =\frac{1}{2 r} \ln \left(\frac{1+r}{1-r}\right) .
\end{aligned}
$$

Further simplification is only possible if the Fresnel reflectivity $r$ is much smaller than one. Then the expansion of the logarithm yields

$$
\begin{aligned}
& \kappa_{0} \Lambda=-2 r \\
& \delta_{1} \Lambda=1 .
\end{aligned}
$$

Thus, only if the conditions $\Delta \phi_{0}=0$ and $r \ll 1$ are fulfilled can we write

$$
\begin{aligned}
& \kappa \Lambda=-2 r \\
& \delta \Lambda=\phi-\pi .
\end{aligned}
$$

Whereas the linear detuning coefficient $(\mathrm{C} 18)$ is the same as in Section II, we see that the constant coupling coefficient (C17) is equal to (5).

\section{REFERENCES}

[1] H. A. Macleod, Thin-Film Optical Filters. Bristol: Adam Hilger, 1985.

[2] J. R. Pierce, "Coupling of modes of propagation," J. Appl. Phys., vol. 25, no. 2, pp. 179-183, Feb. 1954.

[3] M. Matsuhara, K. O. Hill, and A. Watanabe, "Optical-waveguide filters: Synthesis," J. Opt. Soc. Amer., vol. 65, no. 7, pp. 804-809, July 1975.

[4] H. Kogelnik and C. V. Shank, "Coupled-wave theory of distributed feedback lasers," J. Appl. Phys., vol. 43, no. 5, pp. 2327-2335, May 1972.

[5] R. Szipöcs, K. Ferencz, C. Spielmann, and F. Krausz, "Chirped multilayer coatings for broadband dispersion control in femtosecond lasers," Opt. Lett., vol. 19, no. 3, pp. 201-203, Feb. 1994.

[6] L. R. Brovelli and U. Keller, "Simple analytical expressions for the reflectivity and the penetration depth of a Bragg mirror between arbitrary media," Opt. Commun., vol. 116, pp. 343-350, May 1995.

[7] H. A. Haus, Waves and Fields in Optoelectronics. Englewood Cliffs, NJ: Prentice-Hall, 1984.

[8] G. P. Agrawal and N. K. Dutta, Long-Wavelength Semiconductor Lasers. New York: Van Nostrand Reinhold, 1986

[9] M. Born and E. Wolf, Principles of Optics. London, U.K.: Pergamon, 1984.

[10] F. Abelès, "Recherches sur la propagation des ondes électromagnétiques sinusoïdales dans les milieux stratifiés. Application aux couches minces," Ann. de Physique, $12^{e}$ série, t. 5, pp. 706-782, Jan.-Fév. 1950.

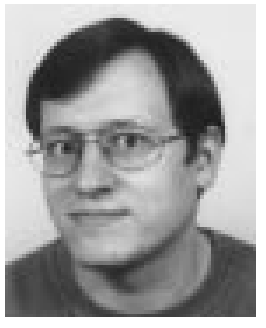

Nicolai Matuschek was born in Biberach/Riß, Germany, 1968. He received the diploma degree in physics from the University Ulm, Germany, in 1995. $\mathrm{He}$ is currently pursuing the $\mathrm{Ph} . \mathrm{D}$. degree at the Institute of Quantum Electronics, Swiss Federal Institute of Technology (ETH) Zürich, Switzerland.

After finishing his diploma degree in the field of theoretical polymer physics, he changed his research interests to laser physics, where he is currently focused on the theory of mode-locked solid-state lasers.

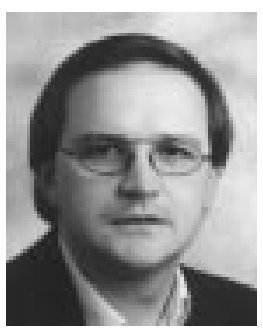

Franz X. Kärtner was born in Cham, Germany, in 1961. He received the Diploma and the Ph.D degrees in electrical engineering from the Technical University of Munich, Munich, Germany, in 1986 and 1989, respectively.

From 1991 to 1993, he was a Feodor-Lynen Research Fellow of the Humboldt Foundation at the Massachusetts Institute of Technology, Cambridge. Since 1993, he has been with the Institute of Quantum Electronics, Swiss Federal Institute of Technology (ETH) Zürich, Switzerland. He carried out research on noise in electronic circuits and optical devices, generation of squeezed states in the microwave and optical domain, quantum optics and laser physics in general. He is currently focused on the dynamics of modelocked lasers.

Dr. Kärtner is a member of the German Physical Society and the Optical Society of America.

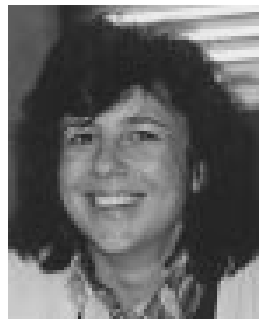

Ursula Keller (M'89) was born in Zug, Switzerland, in 1959. She received the "Diplom" in physics from the Federal Institute of Technology (ETH) Zürich, Switzerland, in 1984 and the M.S. and Ph.D. degrees in applied physics from Stanford University, Stanford, CA, in 1987 and 1989, respectively. Her $\mathrm{Ph} . \mathrm{D}$. research was in optical probing of charge and voltage in GaAs integrated circuits and in low-noise ultrafast laser systems.

From late 1984 to 1985 , she worked on optical bistability at Heriot-Watt University, Edinburgh, Scotland. In 1989, she joined AT\&T Bell Laboratories, Holmdel, NJ, as a Member of Technical Staff, where she conducted research on photonic switching, ultrafast laser systems, and semiconductor spectroscopy. Since March 1993, she has been an Associate Professor in the Physics Department at the Swiss Federal Institute of Technology (ETH) in Zürich, Switzerland. Her current research interests are ultrafast lasers, spectroscopy, local probes, and novel devices for applications in optical information processing, communication, and medicine

Prof. Keller is a member of the Optical Society of America, the European Physical Society, the Swiss Physical Society, and the Swiss Academy of Technical Sciences. During 1985-1986, she was a Fulbright Fellow and in 1987-1988, she received an IBM Predoctoral Fellowship. 